Journal of the Korean Physical Society, Vol. 70, No. 4, February 2017, pp. $339 \sim 347$

\title{
Eigen Solutions, Shannon Entropy and Fisher Information under the Eckart Manning Rosen Potential Model
}

\author{
C. A. Onate \\ Department of Physical Sciences, Landmark University, Omu-Aran, Nigeria \\ M. C. Onyeaju * and A. N. Ikot \\ Theoretical Physics Group, Department of Physics, University of Port Harcourt, Nigeria \\ J. O. A. Idiodi \\ Theoretical/Mathematical Physics Section, Department of Physics, University of Benin, Nigeria \\ J. O. Ojonubah \\ Mathematics Department, Federal College of Education Okene, Nigeria
}

(Received 11 August 2016, in final form 18 November 2016)

Abstract

We solved the Schr" odinger equation with a certain approximation to the centrifugal term for an arbitrary angular momentum state with the Eckart Manning Rosen potential. The bound-state energy eigenvalues and the corresponding wave functions have been approximately obtained using the parametric Nikiforov Uvarov method. The solutions of the Schr" odinger equation for the Eckart potential, Manning Rosen potential, and Hulth' en potential have been obtained using a certain transformation. The concepts of the Shannon entropy and the Fisher information of a system under the Eckart Manning Rosen potential are investigated in detail. The behavior of the screening parameter and the quantum number $\mathrm{n}$ for Fisher information and the Shannon entropy are

also

investigated.

PACS numbers: 03.65.Ge, 03.65.Fd, 03.65.-W, 03.67.-a

Keywords: Eigen solutions, Wave equation, Shannon entropy, Fisher information theoretic quantities

DOI: $10.3938 / \mathrm{jkps} .70 .339$

Available at: www.sringer.com/article. 